\title{
On the Accuracy of Nonlinear Microwave Circuit Simulation at the Behavioral Level
}

\author{
S.L. Loyka ${ }^{1}$
}

\begin{abstract}
Behavioral-level simulation is a widely-used design tool for digital wireless communication systems operating at RF/microwave frequencies. However, success in using this tool depends substantially on the simulation accuracy. In this paper, we consider some factors contributing to the accuracy of behavioral-level simulation. A key factor is an accurate measurement of envelope AM-AM and AM-PM transfer functions and an accurate computation of instantaneous transfer functions. Some practical recommendations how to improve the simulation accuracy are given.
\end{abstract}

\section{INTRODUCTION}

Nonlinear behavior of various microwave circuits may lead to a substantial degradation in the performance of wireless communication systems. For example, the nonlinear performance of power amplifiers has a profound impact on the operation of CDMA communication systems. Several behavioral-level simulation techniques are widely used for the nonlinear simulation of power amplifiers and for their optimization [1]-[3]. However, for this kind of simulation techniques, the simulation accuracy is of primary concern due to the following reasons: (1) the problem under consideration is a nonlinear one (i.e. we must ensure wide dynamic range of the analysis and reasonable accuracy of the analysis at the same time that is not a simple task), and (2) behavioral-level techniques use the single-tone input-output envelope transfer functions (amplitude-to-amplitude (AM-AM) and amplitudeto-phase (AM-PM)) to characterize the nonlinear circuit operation in multiple-tone regime that gives several additional contributions to the entire simulation error. In this paper, we discuss some factors that influence the accuracy of behavioral-level simulation. For our investigation, we consider mainly two behavioral-level techniques: the quadrature modeling technique and the instantaneous quadrature technique [1]-[3].

The main principle of these techniques is to use the AM-AM and AM-PM transfer functions obtained from single-tone measurements to simulate the circuit operation. While the quadrature modeling technique uses the complex envelope of a bandpass RF signal and the envelope transfer functions (AM-AM and AM-PM) of a nonlinear element,

$$
K\left(A_{\text {in }}\right)=A_{\text {out }}\left(A_{\text {in }}\right) / A_{\text {in }}, \quad \Phi\left(A_{\text {in }}\right)=\varphi_{\text {out }}\left(A_{\text {in }}\right)-\varphi_{\text {in }}
$$

where $A_{\text {in }}$ and $\varphi_{\text {in }}$ - are the input signal's amplitude and phase (in the single-tone regime), and $A_{\text {out }}\left(A_{\text {in }}\right)$ and $\varphi_{\text {out }}\left(A_{\text {in }}\right)$ - are the same for the output signal (but now they are functions of the input signal amplitude due to the element's nonlinearity), the instantaneous quadrature technique uses instantaneous values of the RF signal and the instantaneous transfer functions of the nonlinear element. A system of two integral equations relates the envelope and instantaneous transfer factors [3].

\section{IMPACT OF The Transfer FUnCTIONS ON SimUlation ACCURACY}

Thus, the AM-AM and AM-PM transfer functions are the key component of the entire simulation method. When determining these functions (through measurements or circuit-level simulation), one should take into account the following:

1) AM-AM and AM-PM measured (or simulated) data are available for a finite set of points. During simulation, these data are usually required for another set of points. Thus, a mathematical technique is required to transform these data from one set of points to another. Besides, these data must be stored in some form (look-up tables, for example) and that technique can also be used for this purpose. This is a representation problem.

2) While the importance of higher-order derivatives of elements' characteristics for the nonlinear circuitlevel simulation is well-recognized [4]-[5], the importance of higher-order derivatives for the nonlinear behavioral-level simulation is not so well understood [6]. Let us now consider this issue in more details. Measured or circuit-level simulated AM-AM and AM-PM data contains not only the "real" characteristics (without any disturbance), but also measurement (or simulation) noise and distortions due to instruments' (or simulator) noise and inaccuracy. These noise and distortions may severe degrade the entire simulation accuracy due to a nonlinear character of the problem. It can be illustrated this by the following argument. Let us assume that the time-domain transfer function is know with some inaccuracy,

$$
y(x)=F_{0}(x)+\Delta F(x)
$$

${ }^{1}$ Belorussian State University of Informatics and Radioelectronics,

P.Brovki St. 6, Minsk 220027, Belarus, email: sergey.loyka@ieee.org 
where $y$ - is the output voltage, $x$ - is the input voltage, $F_{0}$ - is the "real" transfer function, and $\Delta F-$ is a disturbance. Using (2), we calculate the output signal in the time domain (for given $x(t)$ ) and then transform it to the frequency domain. Obviously, the output spectrum contains two components, the "real" spectrum and the disturbance, due to the linearity of the Fourier transform:

$$
S_{y}=S_{0}+\Delta S, \text { where } S_{0}=\mathbf{F T}\left(F_{0}\right), \Delta S=\mathbf{F T}(\Delta F)
$$

and FT is the Fourier Transform. One expects that the analysis dynamic range is at least $100 \mathrm{~dB}$ [1]. Thus, making a rough estimation, one obtains: $\Delta S<10^{-5} S_{0, \max }$ (where $S_{0, \max }$ is the maximum level in the "real" spectrum) and $\Delta F<10^{-5} F_{0}$. Hence, if we wish to obtain the output spectrum within $100 \mathrm{~dB}$ in a general case, the relative disturbance level of the transfer function must be not higher than $10^{-5}-$ much smaller than a typical measurement error. Fortunately, this consideration says nothing about the specific spectrum of the distortion. For example, if $\Delta F=$ constan $t$, then the output spectrum has no any distortions except at $f=0$. One usually does not consider the DC component in system-level RF/microwave applications and, hence, this spectrum can be considered to be undistorted. The next example is $\partial \Delta F / \partial x=$ cons tan $t$. In this case, we just predict the transfer gain with an error, all nonlinear products are predicted correctly (within the accuracy of the gain prediction - all these components are just scaled by this constant). Thus, the constrains above can be relaxed to, say, $\Delta F<10^{-2} F_{0}$ (one percent accuracy). When $\Delta F$ has non-zero higher derivatives, it may generate nonlinear noise which will disturb the nonlinear products we are looking for (for example, some specific intermodulation products). Thus, it is very important to consider not only the magnitude of the transfer function distortion (error), but also its spectrum (higher-order derivatives): we can afford some higher-level disturbance in the transfer function, but only with a specific spectrum. An efficient way to analyze and to control the spectrum of the distortions is to use a series expansion of the transfer functions (the envelope as well as instantaneous ones). We should note that, usually, $\Delta S$ has a noise-like form of quite a small level, while $S_{0}$ represents required spectrum plus nonlinear distortions. Hence, when $\Delta S$ is smaller than all the spectral components in $S_{0}$ that are under consideration, $\Delta F$ has no much influence on the simulation accuracy. This is the case when all the considered spectral components are of high enough level (in the large-signal regime, for example). $\Delta F$ decreases the simulation accuracy of mainly small-level spectral components.

3) Taking into account the previous item, one should "filter out" the measurement (or simulation) noise of the envelope and instantaneous transfer functions before starting simulation. Series expansion can be used for this purpose. We just decrease the expansion order in order to suppress the higher-order noise (disturbance) components. However, we should not decrease it too much because it gives rise to the approximation error (using this series, we approximate the "real" transfer function with larger error). Obviously, there is some optimum value for the series order (however, as to the best of author's knowledge, there is no any mathematical technique to find this optimum).

\section{APProximation of The Transfer Functions USING SERIES EXPANSION}

In the mathematical language, the problem of transfer function (factor) representation can be formulated as follows. We know transfer functions (AM-AM and AM-PM, or in-phase and quadrature components) for a finite set of points (from measurements or circuit-level simulation),

$$
y_{i}=F\left(x_{i}\right)
$$

where $y_{i}$ is the output amplitude or phase, and $x_{i}$ is the input amplitude, but the simulation usually requires for this function to be defined over a continuous interval (strictly speaking - for another finite set of points, but it is more convenient to consider a continuous interval because we don not know this set in advance),

$$
y=F(x), \quad x \in\left[-x_{\text {max }}, x_{\text {max }}\right]
$$

Thus, we need a method to obtain (5) using (4). Several such methods are available [2], [3], [7] which make use of (1) Splines, (2) Bessel series, (3) Sine/cosine series, (4) Chebyshev polynomial series, (5) Power series, (6) Interpolating polynomials, (7) Evolutionary programming techniques (genetic algorithm and neural networks), (8) Wavelets. Splines are very traditional tool. They usually provide the best accuracy (in terms of transfer function representation) [2], but do not allow one to filter out the measurement noise. Thus, the over-all simulation accuracy is not very good when the measurement noise contribution is substantial [7]. Interpolating polynomials are also widely used for such a problem [8], but, unfortunately, they also do not allow filtering out the measurement noise and their accuracy is not very good. On the contrary, a series expansion gives us a good possibility to filter out the measurement noise by changing the expansion order. The approximation (representation) accuracy can also be quite good if the expansion order is chosen appropriately. When evaluating the expansion coefficients, one should keep in mind the following: 
(1) the number $M$ of the measured points should be large enough in order that these points to represent adequately the continuous function, otherwise large errors in the simulations results are possible. For smooth functions, practical value of $M$ equals to 10-100, for fast-changing functions - to 100-1000. A general rule-ofthumb is that the change in the transfer function for successive points should not be larger than $0.5-3 \mathrm{~dB}$.

(2) $M$ is also of great importance for the expansion coefficient calculation due to the coefficient aliasing effect [10]. In order to get a good accuracy of the expansion coefficients , one must use large enough $M$, such that the coefficient aliasing effect can be neglected. A particular value of $M$ depends on a particular transfer function. A general rule-of-thumb is that $M \geq(3-10) N$.

(3) In general, one should not use a too high-order expansion in order to filter out the measurement noise the lower-order expansion is used, the more measurement noise is filtered out. One should also take into account that too low-order expansions should not be used since the simulation accuracy would be very poor. A particular choice of the expansion order depends on the measurement noise level (measurement accuracy) and on fine details of the transfer function.

Genetic algorithm (GA), which appears to be a very effective tool for many kinds of problems, can be used for reducing the series expansion approximation error and, consequently, the entire simulation error [11]. In general, GA methods differ from conventional ones in that (i) they operate on a group (or population) of trial solutions at the same time, and (i) they use stochastic operators (selection, crossover and mutation) to explore the solution domain in search of an optimal solution [12]. In our case, the fitness function is defined to be the maximum difference between the transfer function and its approximation. Thus, using the Genetic Algorithm we can evaluate the expansion coefficient $a_{k}$ in a new way with two substantial advantages: (i) we minimize the approximation error, and (ii) we still have possibility to filter out the measurement (simulation) noise. In fact, using this method, we build the best approximation on the minimax criterion for a given expansion order. For example, the classical Chebyshev expansion error can be reduced in some cases by 1.5 times by this method [11]. It seems that this method can potentially give the best possible approximation on the minimax criterion. This is very important for our problem because we have to use smaller approximation order (to filter out the measurement noise) and, using the GA method, we still achieve quite a good approximation accuracy.

\section{APProximation ACCURACY AND MeASUREMENT NOISE}

The series expansion methods presented in the preceding section are quite efficient tool for the approximation/representation of nonlinear transfer functions for behavioral-level simulation. However, due to the nonlinear character of the problem, special attention must be paid to the approximation accuracy - even small inaccuracies in the transfer function approximation may result in very large inaccuracies in the final simulation results. The main method to control accuracy of a series expansion is through an appropriate choice of the expansion order $(N)$ : if one whishes to increase accuracy, the expansion order should be increased. Of course, there are some limitation to such increase due to the numerical stability of a computational algorithm. However, usually these limitations are quite large and do not influence substantially on a practical choice of the expansion order (for example, for the Chebyshev expansion this limit is about $150-200$ what is far beyond a practically required expansion order).

There is a more serious limitation to the expansion order - the measurement (or simulation) noise which is always present in measured (simulated) AM-AM and AM-PM data, as shown by Eq. (2). What we need is to approximate $F_{0}(x)$ as accurate as possible and, at the same time, to suppress $\Delta F(x)$ as much as possible. When we consider the "spectrum" of $\Delta F(x)$ (in terms of expansion coefficients $a_{k}$ ), we find that it mainly contains high-order components, the contribution of lower-order components is quite small (of course, their levels depend substantially on a particular scenario). On the contrary, the "spectrum" of $F_{0}(x)$ contains mainly low-order components with higher-order components being much smaller. Thus, when we increase the expansion order, the accuracy of $F_{0}(x)$ approximation increases, but, at the same time, the noise contribution to the approximated characteristic (due to $\Delta F(x)$ ) also increases. The optimum value of the expansion order corresponds approximately to the point where the approximation error and the noise contribution are equal. Unfortunately, as to the best of the authors' knowledge, there is no any mathematical technique to determine the optimal order. A practical method to do this is to increase the expansion order (starting from some small value, say, $N=5 \ldots 10$ ) until the simulation accuracy (for example, the accuracy of intermodulation product prediction) starts to decrease.

It should be pointed out that the simulation noise is usually much smaller than the measured one. Some measures must be taken in order to suppress the latter. One possible solution is to use during the measurements the time averaging option of the network analyzer. However, it does not allow suppressing noise completely. Besides, there is another distortion of the AM-PM data (which is usually measured with higher error) - due to the measurement instrument inaccuracy. Under these circumstances, we can use the series expansion as a tool for suppressing the measurement noise (including the measurement inaccuracy component) by controlling the expansion order. Thus, before transforming AM-AM and AM-PM measured data into the instantaneous transfer factors [3], we employ the series expansion of this data (actually, the in-phase and quadrature transfer functions 
are expanded). For simulated AM-AM and AM-PM data this procedure is not required due to small level of the simulation noise. However, special measures must be taken to insure a high accuracy of the simulated data (for example, high relative accuracy and a large number of frequencies should be set during the HB simulation).

In order to study the influence of the measurement noise on the simulation accuracy, we measured and simulated intermodulation products in a microwave amplifier. Some results are as follows. The simulation accuracy is quite good (the discrepancy between simulation and measurement is in average a few dBs) over a wide dynamic range (in access of $130 \mathrm{~dB}$ ) for the optimum approximation order $N=24$ (even 7 th and 9th order IMPs are predicted quite accurately). However, the dependence of the simulation accuracy on the approximation order is highly non-monotonous: increase in the approximation order over the optimum value results in an accuracy decrease rather than increase. The point $N=24$ corresponds to an optimum value for almost all nonlinear products (the minimum discrepancy); further increase in $N$ yields an increase in the simulation error, especially for higher-order nonlinear products. At the same time, the approximation error decreases monotonically (for $N>15$ ) as $N$ increases. Thus, the dependence of the simulation accuracy on the approximation accuracy is highly non-monotonous: increase in the approximation accuracy does not guarantee an increase in the entire simulation accuracy. We attribute this fact to the measurement noise (and other measurement inaccuracies) impact as well as to the inaccuracies (computational noise etc.) of transforming the envelope transfer functions into the instantaneous ones.

Some other factors contributing to the simulation inaccuracy are the following: (i) the bias decoupling network effect [13] that is not taken into account in a conventional simulation (in principle, this effect can be modeled by the instantaneous quadrature technique [3]), (ii) the difference between static and dynamic AM-AM and AM-PM characteristics [14], (iii) the thermal tone spacing effect [15], (iv) memory feedback effects [14].

\section{REFERENCES}

[1] J. F. Sevic, M. B. Steer, A. M. Pavio, "Nonlinear analysis methods for the simulation of digital wireless communication systems," International Journal of Microwave and Millimeter-Wave Computer-Aided Engineering, vol. 6, No. 3, 1996, pp.197-216.

[2] J. Staudinger, "Applying the Quadrature Modeling Technique to Wireless Power Amplifiers," Microwave Journal, vol. 40, No. 11, Nov. 1997, pp.66-86.

[3] S.L. Loyka, J.R. Mosig, New Behavioral-Level Simulation Technique for RF/Microwave Applications. Part I: Basic Concepts, International Journal of $R F$ and Microwave Computer-Aided Engineering, submitted for publication.

[4] S.A. Maas, How to Model Intermodulation Distortion, in IEEE Microwave Theory Tech. Symp. Dig., Boston, MA, 1991, pp.149-151.

[5] N.B. de Carvalho, J.C. Pedro, Multitone Frequency-Domain Simulation of Nonlinear Cicuits in Large- and Small-Signal Regimes, IEEE Trans. MTT, vol. 46, No. 12, Dec. 1998, pp. 2016-2024.

[6] S.L. Loyka, On the Use of Cann's Model for Nonlinear Behavioral-Level Simulation, IEEE Trans. on Vehicular Technology, 1999, submitted for publication.

[7] S.L. Loyka, J.R. Mosig, New Behavioral-Level Simulation Technique for RF/Microwave Applications. Part II: Approximation of Nonlinear Transfer Functions, to be published.

[8] I.D. Cheremisinov, S.L. Loyka,. V.I. Mordachev, Synthesis of the polynomial model of nonlinear elements based on intermodulation dynamic ranges, Proc. of $3^{\text {rd }}$ Inter. Confer. on Telecommunications in Modern Satellite, Cable and Broadcasting Services (TELSIKS'97), Oct. 8-10, Nis, Yugoslavia, 1997, pp. 519-522.

[9] W.H. Press, B.P. Flanneay, S.A. Teukolsky, W.T. Vetterling, Numerical Recipes in C, Cambridge University Press, 1988.

[10] R.W. Hamming, Numerical Methods for Scientists and Engineers, Dover Publications, New York, 1973.

[11] S.L. Loyka, New Method for Nonlinear Transfer Function Approximation, Electronics Letters, vol. 35, No. $10,13^{\text {th }}$ May 1999, pp.789-791.

[12]D.S. Weile, E. Michielssen, Genetic Algorithm Optimization Applied to Electromagnetics: A Review, IEEE Trans. On Antennas and Propagation, vol. 45, N. 3, pp. 343-353, Mar. 1997.

[13] J. Staudinger, Behavioral Analysis Method Applied to the Design \& Simulation of Linear Power Amplifiers, RAWCON'98 Workshop "Modeling and Simulation of Devices and Circuits for Wireless Communications", Colorado Springs, CO, Aug. 9-12, 1998.

[14] W. Bosch, G. Gatti, Measurement and Simulation of Memory Effects in Predistortion Linearizers, IEEE Trans. on MTT, vol. 37, No. 12, Dec. 1989, pp.1885-1890.

[15]P.M. McIntosh, C.M. Snowden, The Effect of a Variation in Tone Spacing on the Intermodulation Performance of Class A \& Class AB HBT Power Amplifiers, IEEE Int. Symp. on MTT, June 8-13, 1997, Denver, CO. 